\title{
Alleviating the Tower Mechanical Load of Multi-MW Wind Turbines with LQR Control
}

\author{
Yoonsu Nam*, Pham Trung Kien ${ }^{\dagger}$, and Yo-Han La* \\ ${ }^{* \dagger}$ School of Mechanical and Mechatronics Engineering, Kangwon National University, Chuncheon, Korea
}

\begin{abstract}
This paper addresses linear quadratic regulation (LQR) for variable speed variable pitch wind turbines. Because of the inherent nonlinearity of wind turbines, a set of operating conditions is identified and then a LQR controller is designed for each of the operating points. The feedback controller gains are then interpolated linearly to get a control law for the entire operating region. In addition, the aerodynamic torque and effective wind speed are estimated online to get the gain-scheduling variable for implementing the controller. The potential of this method is verified through simulation with the help of MATLAB/Simulink and GH Bladed. The performance and mechanical load when using LQR are also compared with those obtained when using a PI controller.
\end{abstract}

Key words: LQR control, Multi-MW size wind turbine, Variable speed variable pitch wind turbine, Wind energy conversion system

\section{INTRODUCTION}

Recently, variable speed variable pitch wind turbines (VSVP WT) have been continuously increasing their market share. This configuration is the best for multi-megawatt machines because it can maximize the energy captured over a wide range of wind speeds and reduce the mechanical fatigue by using aerodynamics control systems. In this configuration, the turbine operates with a variable speed and a fixed pitch if the wind speed is in below the rated region (region 2) to achieve maximum aerodynamic efficiency. When the wind speed is above the rated region (region 3) the rotor is regulated at its rated speed by varying the pitch angle to ensure that the mechanical limitations are not exceeded. The controller should be designed intelligently to transit smoothly between the two regions and to ensure the other requirements during transition such as limiting bladed-tip noise, minimizing output power fluctuations, etc.

The thrust force acting on the rotor and torque developed by the wind turbine are nonlinear functions of the wind speed, rotor speed and pitch angle. In addition, modern, large-size wind turbines are usually equipped with individual pitch actuators at each blade and force/moment sensors or accelerometers on the tower, nacelle and blades. These inputs

Manuscript received Feb. 12, 2012; revised Aug. 5, 2013

Recommended for publication by Associate Editor Seung-Ho Song.

${ }^{\dagger}$ Corresponding Author: ptkien.ac@gmail.com

Tel: +82-33-253-0840, Fax: +82-33-257-4190, Kangwon University

*School of Mechanical and Mechatronics Engineering Kangwon National University, Korea and outputs combine with the structural modes to make the machine an inherently nonlinear multi-input multi-output (MIMO) system. However, most of the control methods applied to these multi-control-objective MIMO systems are implemented by using multiple single-input single-output (SISO) loops [1]. The basic multiple-SISO-loop structure of a wind turbine system is illustrated in Fig. 1. This configuration has two loops which operate independently of each other. The top part of this figure is the pitch PI control loop which has a major role for regulating the rotor speed in the above rated region. Below the rated wind speed, the blade pitch angle is pitched off at an optimal value so as to maintain max-Cp operation. In addition, in this region, varying the rotor speed proportionally to the wind speed is a function of the torque control loop presented in the bottom part of the figure.

The linear quadratic regulation (LQR) technique has been applied to wind turbines in [2]-[4]. Most of these studies focus on the high wind speed region, using only the drive-train model or considering only the tower vibration mode in the fore-aft direction. The purpose of this work is to design a MIMO LQR controller for a multi-megawatt wind turbine.

The proposed controller has the same strategy for the entire operating region. The controller is synthesized with the objective of reaching a trade-off between maximizing the energy captured from the wind and mitigating the mechanical load of tower in both the fore-aft and side-to-side directions. Because of the nonlinearity of wind turbines, the controller is designed for specific operating points. The feedback gains are then interpolated or extrapolated for the whole operating 


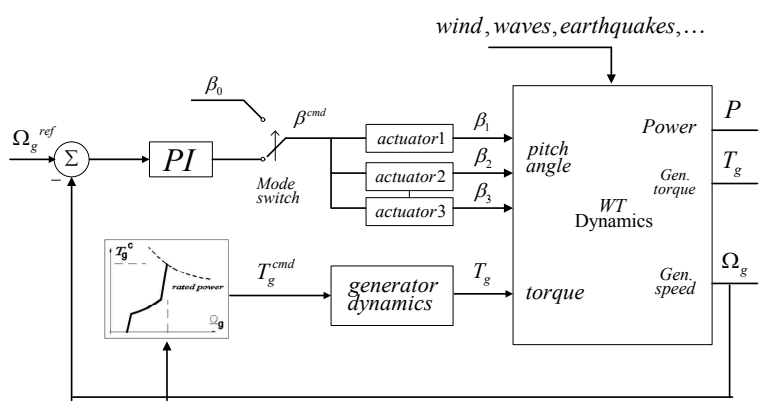

Fig. 1. Control loop of a MW Wind turbine.

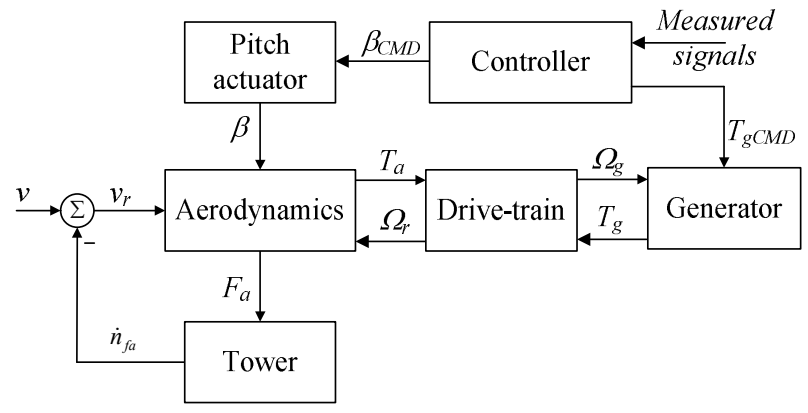

Fig. 2. A block diagram for VSVP WT.

region. The potential of the LQR controller is verified by the commercial wind turbine simulation package GH Bladed, and compared with a PI controller.

This paper is presented as follows. Section II presents the nonlinear dynamics of the wind turbine. Section III derives a linear model for control design and to develop the linear quadratic optimal control which aims for a trade-off between the control objectives. Finally, in section IV, the proposed controller is illustrated in a high-fidelity simulation environment on a representative $2 \mathrm{MW}$ wind turbine.

\section{WIND TURBINE MODEL}

Fig. 2 shows a basic block diagram for the entire variable speed variable pitch wind energy conversion system which can be structured as several interconnected subsystems. In that figure, $F_{a}$ is the thrust force, $\beta$ is the blade pitch angle, and $T_{a}$ and $T_{g}$ are the aerodynamic torque and the reaction generator torque, respectively. $\Omega_{r}$ and $\Omega_{g}$ are the rotor speed and the generator speed, $v$ is the wind speed which is described by the mean wind speed $v_{m}$, and turbulent wind $v_{t}$, is given by (1).

$$
v=v_{m}+v_{t}
$$

The wind speed $v_{r}$ seen by the rotor plane is defined by (2), where $n_{f a}$ is the nacelle displacement in the fore-aft direction.

$$
v_{r}=v-\dot{n}_{f a}
$$

\section{A. Aerodynamic Conversion}

The thrust force acting on the entire rotor, the useful torque developed by the wind turbine and the aerodynamic power captured from the wind are expressed by the highly nonlinear equations as follows [5]:

$$
\begin{aligned}
& F_{a}=\frac{1}{2} \rho \pi R^{2} C_{T}(\lambda, \beta) v_{r}^{2} \\
& T_{a}=\frac{1}{2} \rho \pi R^{3} \frac{C_{P}(\lambda, \beta)}{\lambda} v_{r}^{2} \\
& P_{a}=\frac{1}{2} \rho \pi R^{2} C_{P}(\lambda, \beta) v_{r}^{3}
\end{aligned}
$$

where $\rho$ is the air density, $R$ is the rotor radius, $C_{T}$ is the force coefficient, and $C_{P}$ is called the power coefficient which represents the wind turbine power conversion efficiency. $C_{T}$ and $C_{P}$ are functions of the blade pitch angle and the tip-speed ratio, $\lambda$, defined by:

$$
\lambda=R \Omega_{r} / v_{r}
$$

The coefficients $C_{T}$ and $C_{P}$ are very important in terms of the turbine control system design. The characteristics for different values of the tip-speed ratio and the pitch angle are illustrated in Fig. 3 and Fig. 4. Fig. 3 indicates that there is one specific set of $\lambda$ and $\beta$ where the turbine power coefficient is at its maximum, $\mathrm{C}_{\mathrm{P}}$ max. This means that if the pitch angle is fixed at the optimal value $\beta_{0}$ and the rotor speed is varied proportionally to wind speed to keep tip-speed ratio at $\lambda_{0}$, where $C_{P}$ coefficient is at its maximum, the wind turbine will extract the maximum power from the wind. However, this operation condition occurs only in the low wind speed region. When the wind speed is high, the tip-speed ratio and the pitch angle are not at their optimal values to ensure that the power and rotor speed are being regulated around their rated values. This is done in order to avoid excessive electrical and mechanical stresses.

\section{B. Drive-train Dynamics}

The drive-train system can be modeled as two inertias interconnected by a spring-damper and a gearbox, as schematically represented in Fig. 5 [7]. The rotor of the wind turbine is mounted at the low speed side of the drive-train system. On the high speed side, the generator is mounted giving the opportunity to control the reaction torque from the generator. The dynamic model of the drive-train system is modeled in the form of equations (7) and (8). In these equations, $\Omega_{r}$ and $\Omega_{g}$ are the rotor speed and generator speed, respectively, $J_{r}$ and $J_{g}$ are the rotor inertia and generator inertia, respectively, $B_{r}$ and $B_{g}$ are the damping of the low speed shaft and the high speed shaft, respectively, and $k_{s}$ and $c_{s}$ are the torsional stiffness and torsional damping of the drive-train axis. 


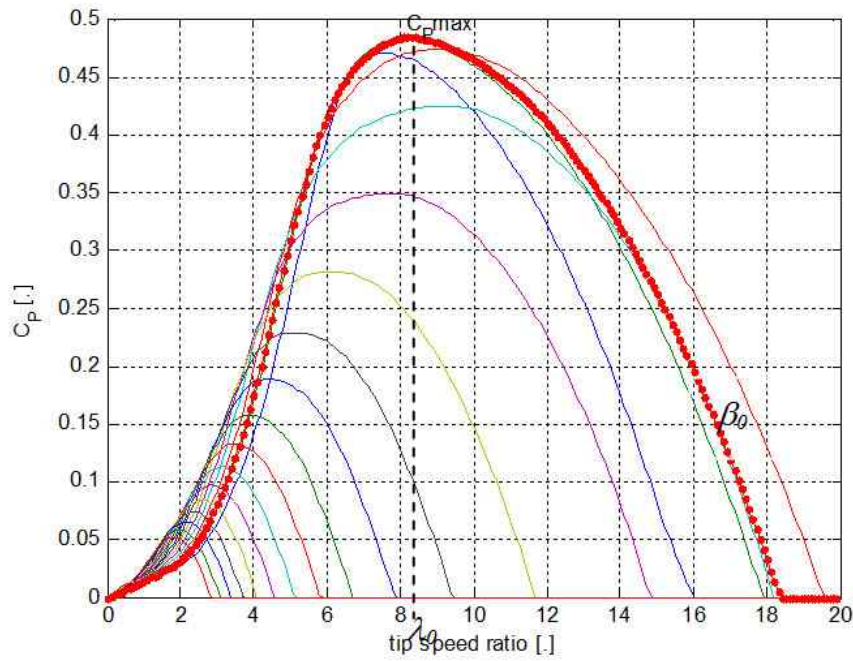

Fig. 3. Power coefficient CP.

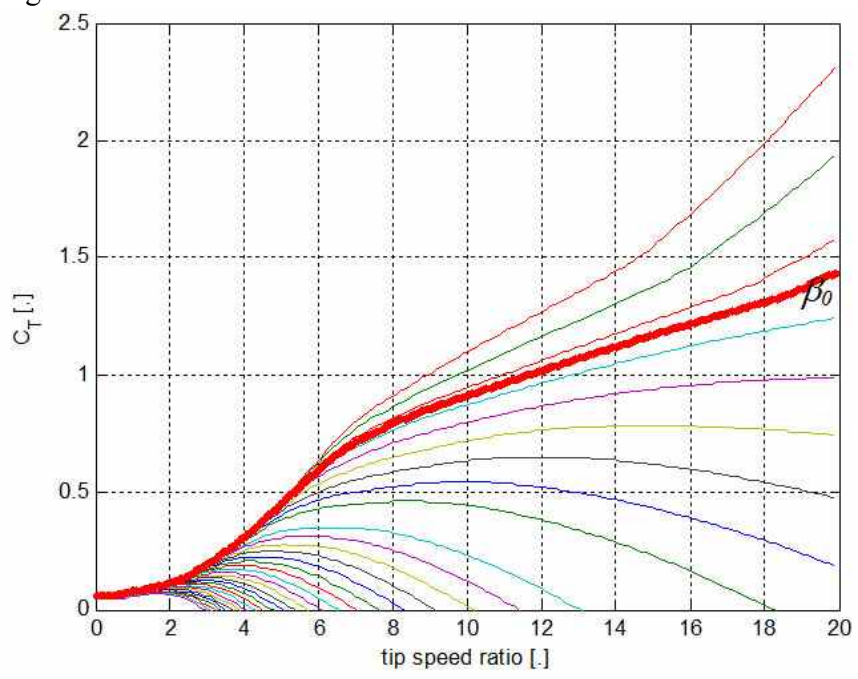

Fig. 4. Force coefficient CT.

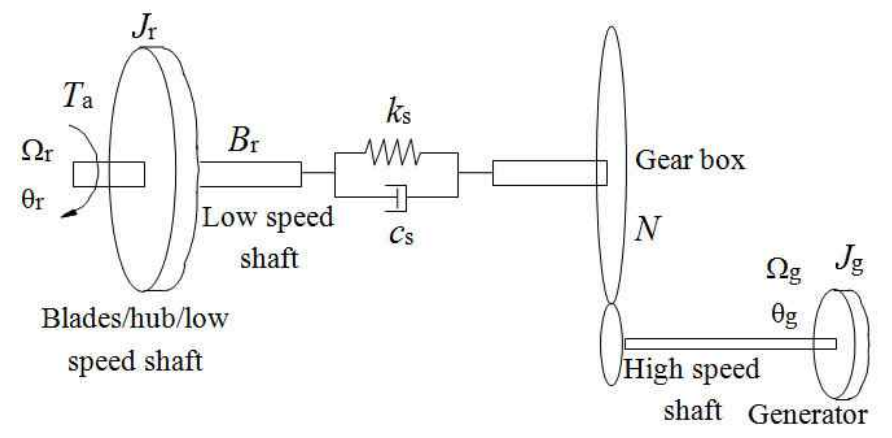

Fig. 5. A drive-train model.

$$
\begin{aligned}
& J_{r} \dot{\Omega}_{r}=T_{a}-k_{s}\left(\theta_{r}-\frac{1}{N} \theta_{g}\right)-c_{s}\left(\Omega_{r}-\frac{1}{N} \Omega_{g}\right)-B_{r} \Omega_{r} \\
& J_{g} \dot{\Omega}_{g}=\frac{k_{s}}{N}\left(\theta_{r}-\frac{1}{N} \theta_{g}\right)+\frac{c_{s}}{N}\left(\Omega_{r}-\frac{1}{N} \Omega_{g}\right)-B_{g} \Omega_{g}-T_{g}
\end{aligned}
$$

The gearbox ratio $N$ is defined as $N=\Omega_{g} / \Omega_{r}$.

\section{Tower Dynamics}

In order to simply get a sufficient dynamic model of the tower for designing the controller in this paper, the first bending mode in both the fore-aft direction and side-to-side direction are considered. When neglecting the tower top rotation, torsion deformation, yawing effects, higher bending modes and tilt influences, the tower top displacements of the first bending mode in both directions can be modeled by two common mass-spring-damper systems as in (9) and (10) [5]. It is also noted that the effects of the sideward aerodynamic fore on the sideward vibration of the tower can be neglected because it is much smaller than the reaction torque of the nacelle.

$$
\begin{gathered}
M_{T} \ddot{n}_{f a}+C_{T} \dot{n}_{f a}+K_{T} n_{f a}=F_{a}-\frac{3}{2 h} M_{t i l t} \\
M_{T} \ddot{n}_{s s}+C_{T} \dot{n}_{s s}+K_{T} n_{s s}=\frac{3}{2 h} T_{n a c}
\end{gathered}
$$

$M_{T}$ is the tower top effective mass, which is determined by the sum of the nacelle mass including the rotor and the top equivalent tower mass. $C_{T}$ and $K_{T}$ are the structural damping and bending stiffness, respectively. $n_{f a}, \dot{n}_{f a}$ and $\ddot{n}_{f a}$ are the tower top displacement, velocity and acceleration in the fore-aft direction, respectively. $n_{s s}, \dot{n}_{s s}$ and $\ddot{n}_{s s}$ represent these values in the side-to-side direction. $h$ is the hub height. The reaction torque of the nacelle, $T_{\text {nac }}$, is given by:

$$
T_{n a c}=\frac{N-1}{N} J_{g} \dot{\Omega}_{g}+T_{g}
$$

\section{Generator Dynamics}

The generator torque is controlled by a power converter which also manages the active and reactive powers of the generator. Because the high speed switching power electronics are able to set the electric generator torque almost instantaneously with respect to the mechanical dynamics, the generator dynamics are sufficiently modeled by the first order transfer function presented in (12):

$$
\frac{T_{g}(s)}{T_{g_{C M D}}(s)}=\frac{1}{1+\tau_{g} s}
$$

In the above equation, $T_{g}(s)$ and $T_{g C M D}(s)$ are the generator torque and generator torque commands, respectively. $\tau_{g}$ is the time constant of the generator dynamics.

\section{E. Pitch Actuator}

When wind turbine systems operates in the high wind speed region, a high rotor speed that may cause mechanical damage which can no longer be managed by increasing the generated power because this would lead to overloading the generator and converter [4]. As seen in Fig. 3, $C_{P}$ can be reduced by varying the pitch angle $\beta$ to maintain the output power at its rated power and to regulate the rotor speed at its rated value, instead of changing the rotor speed proportionally to the wind speed as in the low wind speed 


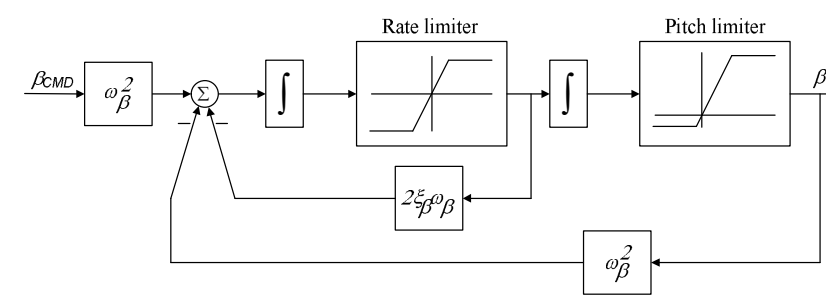

Fig. 6. A second order pitch actuator model.

region.

The pitch actuator is a nonlinear servo that generally rotates all of the blades or part of them in unison. In a closed loop the pitch actuator can be modeled as a linear low-order dynamic system with saturation in the amplitude and derivative of the output signal. Fig. 6 shows a block diagram of a second-order actuator model. The dynamic behavior of a pitch actuator operating in its linear region is described by the following transfer function:

$$
\frac{\beta(s)}{\beta_{C M D}(s)}=\frac{\omega_{\beta}^{2}}{s^{2}+2 \xi_{\beta} \omega_{\beta}+\omega_{\beta}^{2}}
$$

where $\beta$ and $\beta_{C M D}$ are the actual and desired pitch angles, respectively. $\omega_{\beta}$ and $\xi_{\beta}$ are the natural frequency and damping ratio of the pitch actuator dynamics, respectively. For a multi-MW wind turbine, the pitch angle ranges from $-3^{\circ}$ to $90^{\circ}$ and varies at a maximum rate of $\pm 10^{\circ} / \mathrm{s}$.

\section{CONTROLLER DESIGN}

\section{A. Model for the Controller Design}

Equations (7) through (13), describing the dynamics of a wind turbine, can be written in compact form as:

$$
\left\{\begin{array}{l}
f(x, \dot{x}, u, v, t)=0 \\
y=g(x)
\end{array}\right.
$$

By defining the state, input and output vectors:

$$
\begin{aligned}
& x=\left[\begin{array}{lllllllllll}
n_{f a} & \dot{n}_{f a} & n_{s s} & \dot{n}_{s s} & \beta & \dot{\beta} & \Omega_{r} & \theta_{r} & \Omega_{g} & \theta_{g} & T_{g}
\end{array}\right]^{T} \\
& u=\left[\begin{array}{ll}
\beta_{C M D} & T_{g C M D}
\end{array}\right]^{T} ; y=\Omega,
\end{aligned}
$$

Equation (14) is a highly nonlinear model, due to the expression of the extracted thrust force $F_{a}$ and the aerodynamic torque $T_{a}$ as in (3) and (4). In order to design the controller, the global model can be linearized around the operating points by linearizing the aerodynamic torque and thrust force. The deviations of the aerodynamic torque and thrust force from the steady state values are expressed in (14).

$$
\begin{aligned}
& \delta T_{a}=k_{\Omega_{r}}^{T_{a}} \delta \Omega_{r}+k_{\beta}^{T_{a}} \delta \beta+k_{v_{r}}^{T_{a}} \delta v_{r} \\
& \delta F_{a}=k_{\Omega_{r}}^{F_{a}} \delta \Omega_{r}+k_{\beta}^{F_{a}} \delta \beta+k_{v_{r}}^{F_{a}} \delta v_{r}
\end{aligned}
$$

where the operator $\delta$ corresponds to the deviation of the values from the linearization point $\mathrm{OP}(\mathrm{x} 0, \mathrm{u} 0, \mathrm{v} 0)$, and the coefficients in the above equations are defined by:

$$
\begin{aligned}
& k_{\Omega_{r}}^{T_{a}}=\partial T_{a} /\left.\partial \Omega_{r}\right|_{O P} ; k_{\beta}^{T_{a}}=\partial T_{a} /\left.\partial \beta\right|_{O P} ; k_{v_{r}}^{T_{a}}=\partial T_{a} /\left.\partial v_{r}\right|_{O P} \\
& k_{\Omega_{r}}^{F_{a}}=\partial F_{a} /\left.\partial \Omega_{r}\right|_{O P} ; k_{\beta}^{F_{a}}=\partial F_{a} /\left.\partial \beta\right|_{O P} ; k_{v_{r}}^{F_{a}}=\partial F_{a} /\left.\partial v_{r}\right|_{O P}
\end{aligned}
$$

Thus, the wind parameterized linear model of the wind turbine around an operating point can be set on the state space representation as in (18):

$$
\left\{\begin{array}{l}
\delta \dot{x}=A\left(v_{0}\right) \delta x+B\left(v_{0}\right) \delta u+B_{v}\left(v_{0}\right) \delta v_{r} \\
\delta y=C \delta x
\end{array}\right.
$$

where $\delta x=x-x_{0} ; \delta u=u-u_{0} ; \delta y=y-y_{0}$.

The state space matrices $\mathrm{A}(\mathrm{v} 0), \mathrm{B}(\mathrm{v} 0)$ and $\mathrm{B}_{v}(\mathrm{v} 0)$ depend on the operating points.

$$
\begin{aligned}
& A\left(v_{0}\right)=\partial f /\left.\partial x\right|_{O P} ; B\left(v_{0}\right)=\partial f /\left.\partial u\right|_{O P} ; B_{v}\left(v_{0}\right)=\partial f /\left.\partial v_{r}\right|_{O P} \\
& C=\left[\begin{array}{lllllllllll}
0 & 0 & 0 & 0 & 0 & 0 & 1 & 0 & 0 & 0 & 0
\end{array}\right]
\end{aligned}
$$

\section{B. Target Trajectory}

As mention above, when the wind speed is below the rated region, the blade pitch angle is constantly maintained at its optimal value $\beta_{0}$. The rotor speed is changed proportional to the wind speed so as to maintain the tip speed ratio at a constant value $\lambda_{0}$. On the other hand, above the rated region, the pitch angle is varied in order to regulate the rotor speed and to generator torque/power at the rated values. Fig. 7 illustrates the goal trajectories for rotor speed (the first window), blade pitch angle (the second window), generator torque (the third window) and electrical power (the fourth window).

\section{LQR Design}

With the operating points determined, a set of controllers can be synthesized by applying a LQR for the linear model presented in (18), with a quadratic cost function for the regulation problem at an operating point defined as:

$$
J=\int_{0}^{\infty}\left(\delta x^{T} Q\left(v_{0}\right) \delta x+\delta u^{T} R\left(v_{0}\right) \delta u\right) d t
$$

where $\mathrm{Q}\left(v_{0}\right)$ and $\mathrm{R}\left(v_{0}\right)$ are nonnegative and symmetric matrices of the weights. The control law optimizing the above criterion $J$, is a state feedback law with an optimal gain matrix determined by solving an LQR problem. In the implementation, the intermediate controllers are then interpolated linearly from the discrete number of controllers, which have been designed for specific operating points. Fig. 8 illustrates the structure of the wind turbine system. The LQR feedback gains are scheduled by the effective wind speed, and estimated by a wind speed estimator. This estimation will be presented in the following subsection. The drive-train vibration is damped by a drive-train damper. 

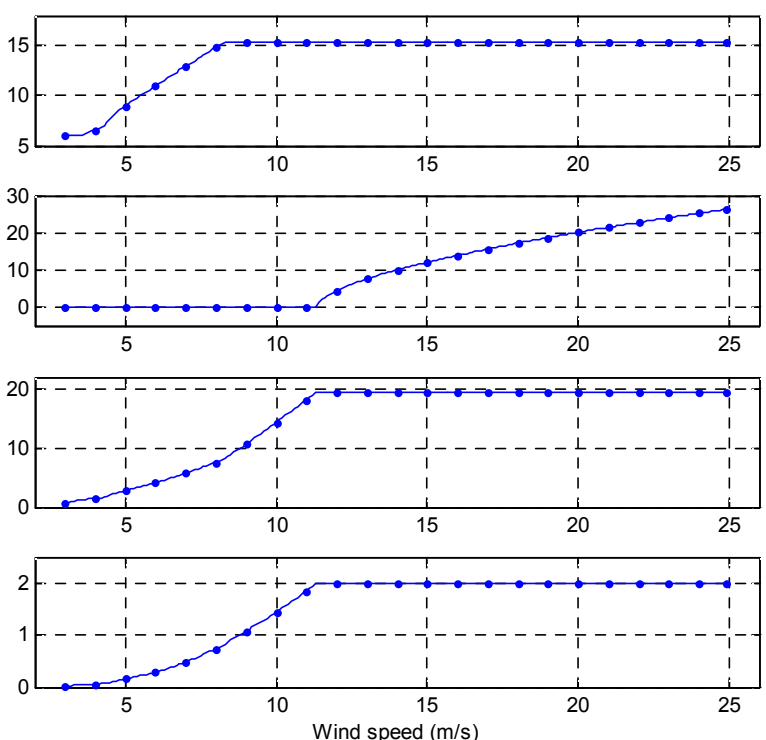

Fig. 7. Schedule for regulation set point.

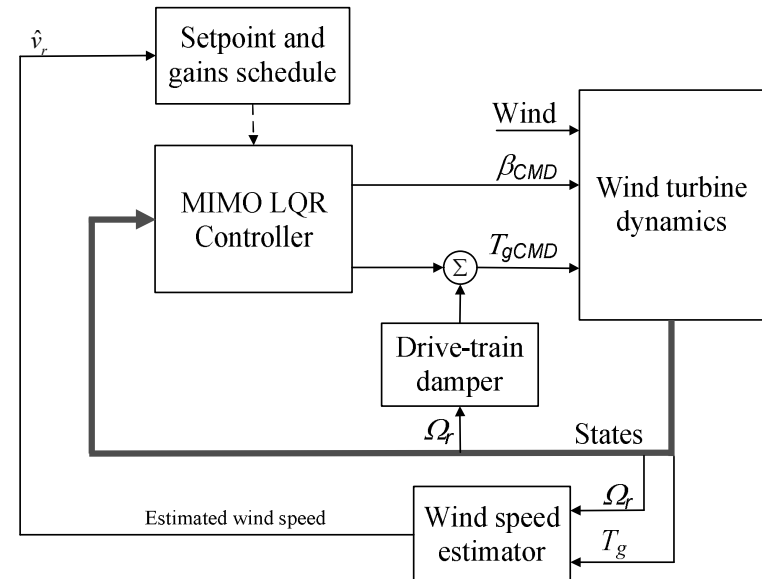

Fig. 8. LQR control system.

\section{WIND SPEED ESTIMATION}

As explained above, the target trajectories are scheduled by the wind speed. However, there is a very rough measurement of the wind speed available. Therefore, it is necessary to have a good wind speed estimator to get the controllers implementable. Fig. 9 shows a schematic diagram of a wind speed estimator, which consists of three consecutive processing modules. The first module is the aerodynamic torque estimation, following by a 3-D lookup table to calculate the effective wind speed, and the next is a low pass filter [6].

The governing equations of the drive-train model (7) and (8) can be combined into one equation as:

$$
J_{t} \frac{d \Omega_{r}}{d t}=T_{a}-N T_{g}-T_{L}
$$

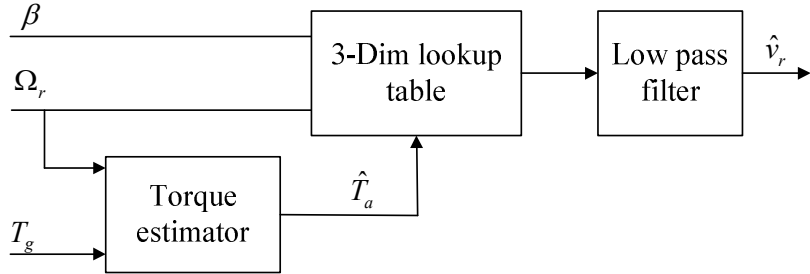

Fig. 9. Wind speed estimator.

where $J_{t}=J_{r}+N^{2} J_{g}$, and $T_{L}$ represent all of the mechanical losses.

Then, augmenting (21) with the unknown aerodynamic torque Ta, the above equation can be expressed in the state space form of (22) [6], [7]:

$$
\begin{aligned}
& \dot{x}=\left\{\begin{array}{c}
\dot{\Omega}_{r} \\
\dot{T}_{a}
\end{array}\right\}=\left[\begin{array}{cc}
0 & 1 / J_{t} \\
0 & 0
\end{array}\right]\left\{\begin{array}{c}
\Omega_{r} \\
T_{a}
\end{array}\right\} \\
&+\left(\begin{array}{c}
-N / J_{t} \\
0
\end{array}\right) T_{g}+\left(\begin{array}{c}
-1 / J_{t} \\
0
\end{array}\right) T_{L}+\left(\begin{array}{c}
-N / J_{t} \\
N
\end{array}\right) w_{g} \\
& y=\left[\begin{array}{ll}
1 & 0
\end{array}\right]\left\{\begin{array}{c}
\Omega_{r} \\
T_{a}
\end{array}\right\}+w_{v}
\end{aligned}
$$

where $w_{g}$ and $w_{v}$ represent the input process and the output sensor noise. A Kalman filter is applied to estimate the aerodynamic torque, which has the structure of (23) where the variables with a hat are to be estimated and $L$ is the Kalman filter gain.

$$
\begin{aligned}
\dot{\hat{x}}=\left\{\begin{array}{c}
\dot{\hat{\Omega}}_{r} \\
\dot{\hat{T}}_{a}
\end{array}\right\}= & {\left[\begin{array}{cc}
0 & 1 / J_{t} \\
0 & 0
\end{array}\right]\left\{\begin{array}{c}
\hat{\Omega}_{r} \\
\hat{T}_{a}
\end{array}\right\} } \\
& +\left(\begin{array}{c}
-N / J_{t} \\
0
\end{array}\right) T_{g}+\left(\begin{array}{c}
-1 / J_{t} \\
0
\end{array}\right) T_{L}+L\left(\Omega_{r}-\hat{\Omega}_{r}\right)
\end{aligned}
$$

The second stage of the wind speed estimation is to calculate wind speed through a 3-D lookup table, which has three inputs. These inputs are the estimated aerodynamic torque, measured rotor speed and measured pitch angle. The 3-D lookup table is build by using equation (4), which is reformulated in the form of:

$$
\hat{T}_{a}=\frac{1}{2} \rho \pi R^{3}\left\{\frac{C_{P}(\hat{\lambda}, \beta)}{\hat{\lambda}}\right\} \hat{v}_{r}^{2}
$$

where $\hat{\lambda}\left(=R \Omega_{r} / \hat{v}\right)$ is the estimated tip-speed ratio.

Fig. 10 illustrates the estimated aerodynamic torque and the estimated wind speed with a mean value of $8 \mathrm{~m} / \mathrm{s}$ and a turbulence intensity (TI) of $23.2 \%$. The real signals and the estimated values are presented in separate windows. As shown in the fourth window of Fig. 10, the estimated wind speed looks like a spatially average wind speed, which is different than the wind speed measured at the hub (dash-dot line). The high frequency components in the turbulent wind 
TABLE I

Performance Data AT Wind Speed of 8M/S Ti $23.2 \%$

\begin{tabular}{|c|c|c|c|c|}
\hline \multirow{2}{*}{$\begin{array}{c}\text { Performance } \\
\text { data }\end{array}$} & \multicolumn{2}{|c|}{ Rotor speed(rpm) } & \multicolumn{2}{c|}{ Elec. power(MW) } \\
\cline { 2 - 5 } & mean & std. & mean & std. \\
\hline PI(A) & 13.7737 & 1.5099 & 0.7410 & 0.2590 \\
\hline LQR(B) & 13.9293 & 1.5936 & 0.7406 & 0.2608 \\
\hline (B-A)/A [\%] & 1.1296 & 5.5434 & -0.0539 & 0.6949 \\
\hline
\end{tabular}

are filtered out to get the estimated wind speed as a good scheduling input variable.

\section{NUMERICAL SimULATION}

The proposed controller is validated through simulations with stochastic wind input in the entire operating region. The performance and mechanical load of proposed method are compared with those of a PI controller. The control law was developed in MATLAB/Simulink and compiled into a Dynamic Link Library (DLL). The external controller in the DLL format will be used with the GH Bladed package to perform a full system simulation of the wind turbine.

A typical example for below the rated region is performed for a wind speed of $8 \mathrm{~m} / \mathrm{s}$, perturbed by a turbulence intensity of $23.2 \%$. The trace of hub height wind speed is shown in the top window of Fig. 11. Fig. 11 also shows the rotor speed behavior, pitch response, generator torque, electric power, tower side-to-side moment $\left(\mathrm{Mx}_{\mathrm{T}}\right)$ and fore-aft moment $\left(\mathrm{My}_{\mathrm{T}}\right)$ versus time. The solid lines in Fig. 11 represent the responses of the wind turbine when using the LQR controller, while the dash-dot lines are the wind turbine responses when using a PI controller (as designed in [6]). The final two plots are the tower fore-aft bending moments $\left(\mathrm{My}_{\mathrm{T}}\right)$ for the LQR and PI controls, respectively. By applying the LQR control, the peak-to-peak magnitude of $\left(\mathrm{My}_{\mathrm{T}}\right)$ decreases a lot. The performance differences of these two responses between the two controllers are summarized in Table I. As shown, the rotor rpm of the LQR control is a little more oscillatory than that of the PI control, but the output powers of two cases are almost the same.

It is difficult to differentiate between the structural responses such as the blade or tower bending moment in the time domain. The damage equivalent loads (DEL), $M_{e q}$, can be a quantitative measure for this ambiguous situation. $M_{e q}$ is given by (25):

$$
M_{e q}=\left(\frac{\sum_{k}\left(M_{k}\right)^{m} n_{k}}{n_{t o t}}\right)^{\frac{1}{m}}
$$

where $n_{k}$ is the number of cycles in the mechanical load range $M_{k}$ and $n_{t o t}$ is the total number of cycles in the mechanical

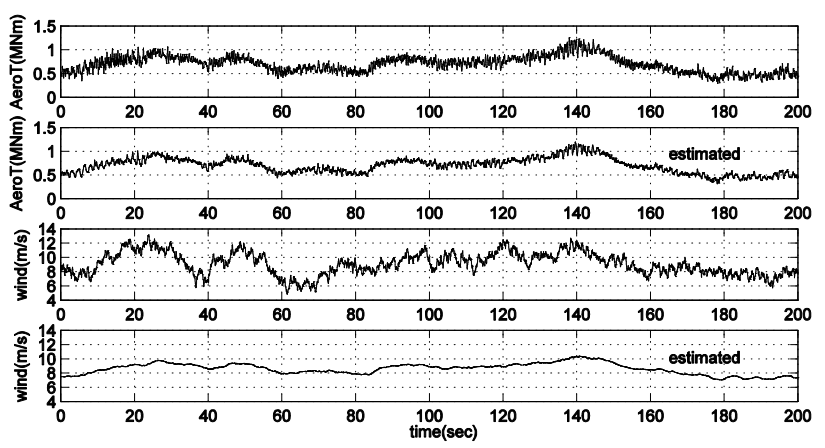

Fig. 10. Estimated aerodynamic torque and wind speed.
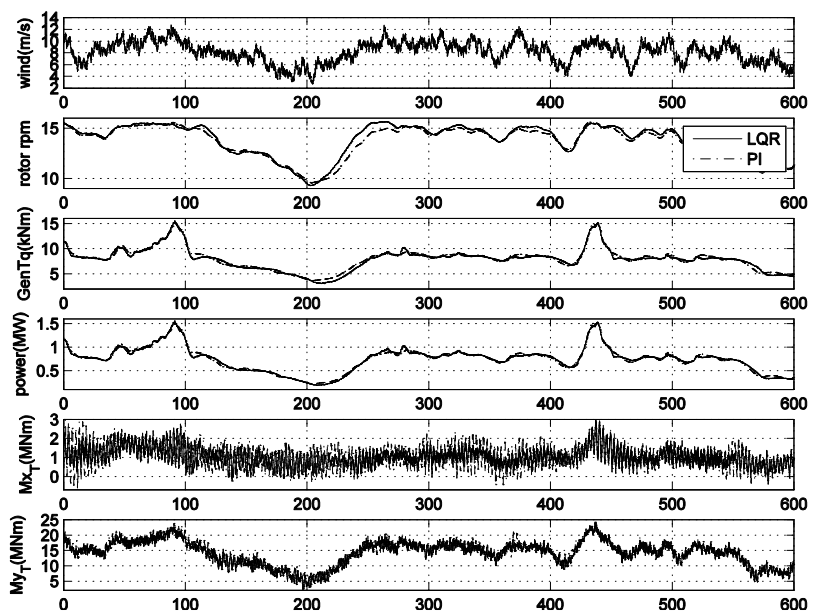

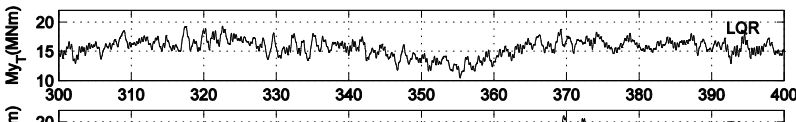

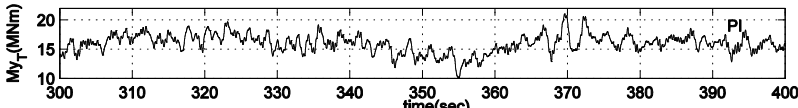

Fig. 11. Simulation result for wind speed of $8 \mathrm{~m} / \mathrm{s} 23.2 \%$ TI.

load signal. $m$ is a material specific number, for example $m=$ 3.5 for a steel tower structure and $m=10$ for a fiber glass blade [10]. The larger the DEL value, the more prone it is to fatigue failure. The statistics for the DEL data of the structural load responses at a mean wind speed of $8 \mathrm{~m} / \mathrm{s}$ for both the LQR and PI controllers are summarized and compared in Table II. The DEL of the blade bending moment in the in-plane $\left(\mathrm{Mx}_{\mathrm{B}}\right)$ direction as well as the tower root bending moment in the fore-aft $\left(\mathrm{My}_{\mathrm{T}}\right)$ and side-to-side direction $\left(\mathrm{Mx}_{\mathrm{T}}\right)$ are significantly improved.

Fig. 12 shows the time domain response simulation for the above rated wind speed region. The wind speed in this simulation has a mean value of $18 \mathrm{~m} / \mathrm{s}$ with a TI of $16.97 \%$. The first window of Fig. 12 shows the wind speed measured at the hub. The second window shows the rotor speed. The next windows gives the pitch, generator torque, and electric power. Again, the solid lines are for the LQR, and the dash-dot lines are for the PI control. Fig. 13 illustrates the in-plane blade root bending moment $\left(\mathrm{Mx}_{\mathrm{B}}\right.$, the first window), 
TABLE II

Mechanical LOAD AT Wind SPEED OF 8M/S Ti 23.2\%

\begin{tabular}{|c|c|c|c|c|}
\hline \multirow{2}{*}{$\begin{array}{c}\text { Mechanical } \\
\text { load }\end{array}$} & \multicolumn{2}{|c|}{$\begin{array}{c}\text { Blade root bending } \\
\text { moment(kNm) }\end{array}$} & \multicolumn{2}{c|}{$\begin{array}{c}\text { Tower root bending } \\
\text { moment }(\mathrm{kNm})\end{array}$} \\
\cline { 2 - 5 } & $\mathrm{Mx}$ & $\mathrm{My}$ & $\mathrm{Mx}$ & $\mathrm{My}$ \\
\hline $\mathrm{PI}(\mathrm{A})$ & 2107 & 1631 & 1274 & 4527 \\
\hline $\mathrm{LQR}(\mathrm{B})$ & 2055 & 1639 & 939.422 & 4262 \\
\hline$(\mathrm{B}-\mathrm{A}) / \mathrm{A}[\%]$ & -2.468 & 0.490 & -26.262 & -5.853 \\
\hline
\end{tabular}

TABLE III

Performance AT Wind SPEed of 18M/S Ti $16.97 \%$

\begin{tabular}{|c|c|c|c|c|}
\hline $\begin{array}{c}\text { Performance } \\
\text { data }\end{array}$ & \multicolumn{2}{|c|}{ Rotor speed(rpm) } & \multicolumn{2}{c|}{ Elec. power(Mw) } \\
\hline & mean & std. & mean & std. \\
\hline PI(A) & 15.2757 & 0.36213 & 1.9920 & 0.0252 \\
\hline LQR(B) & 15.3064 & 0.3783 & 1.9901 & 0.0224 \\
\hline$(\mathrm{B}-\mathrm{A}) / \mathrm{A}[\%]$ & 0.2009 & 4.4652 & -0.0953 & -11.111 \\
\hline
\end{tabular}

TABLE IV

MechanicAl LoAD AT Wind SPEED OF 18M/S Ti 16.97\%

\begin{tabular}{|c|c|c|c|c|}
\hline \multirow{2}{*}{$\begin{array}{c}\text { Mechanical } \\
\text { load }\end{array}$} & \multicolumn{2}{|c|}{$\begin{array}{c}\text { Blade root bending } \\
\text { moment }(\mathrm{kNm})\end{array}$} & \multicolumn{2}{c|}{$\begin{array}{c}\text { Tower root bending } \\
\text { moment(kNm) }\end{array}$} \\
\cline { 2 - 5 } & $\mathrm{Mx}$ & $\mathrm{My}$ & $\mathrm{Mx}$ & $\mathrm{My}$ \\
\hline $\mathrm{PI}(\mathrm{A})$ & 2371 & 2505 & 3702 & 7378 \\
\hline $\mathrm{LQR}(\mathrm{B})$ & 2390 & 2604 & 3378 & 6311 \\
\hline (B-A)/A[\%] & 0.8013 & 3.950 & -8.752 & -14.462 \\
\hline
\end{tabular}

the out-of-plane blade root bending moment $\left(\mathrm{My}_{\mathrm{B}}\right.$, the second window), the tower root bending moment in the sideward direction $\left(\mathrm{Mx}_{\mathrm{T}}\right.$, the third) and the tower root bending fore-aft moment $\left(\mathrm{My}_{\mathrm{T}}\right.$, the last one). The final two windows are enlarged plots for $\mathrm{My}_{\mathrm{T}}$ during the 100 second time period. These are prepared to compare the effectiveness of the LQR control with the PI control. As can be seen, the fore-aft tower root bending moment $\left(\mathrm{My}_{\mathrm{T}}\right)$ for the LQR control becomes much smaller than that of the PI control. The differences in performance and mechanical stresses between the two controllers are summarized in Table III and IV. It can be seen in Table III, that the LQR has a slight decrease in electrical power. However, the power fluctuations decrease remarkably. Furthermore, there is more than a $14 \%$ DEL decrease in the tower bending moment in the fore-aft direction and nearly $9 \%$ in the sideward direction, as shown in Table IV.

The statistical data for the electrical power and tower mechanical loads in the entire operating region are presented in Fig. 14, 15 and 16. It is noted that the simulation at a wind speed of $7 \mathrm{~m} / \mathrm{s}$ has been done with a very high turbulence intensity so that it covers all of the low wind speed region in the operating region of the wind turbine. Although the amount of power captured slightly decreases, the mechanical loads of the tower in both the fore-aft direction and
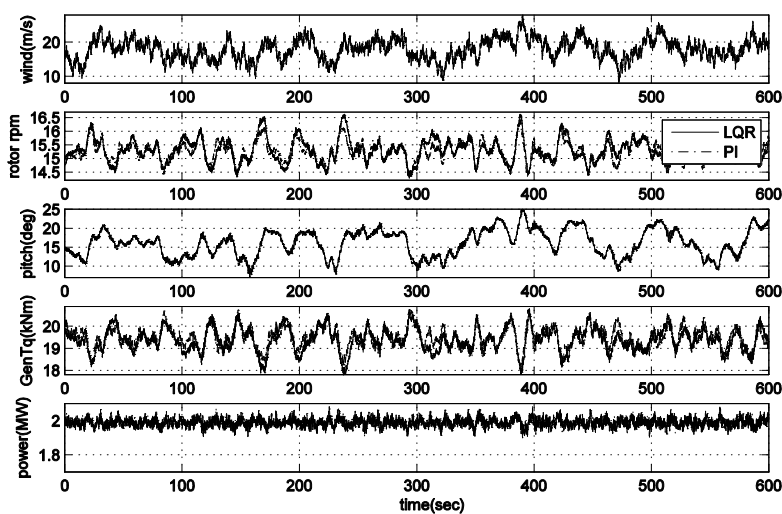

Fig. 12. Simulation result for wind speed of $18 \mathrm{~m} / \mathrm{s} 16.97 \%$ TI.
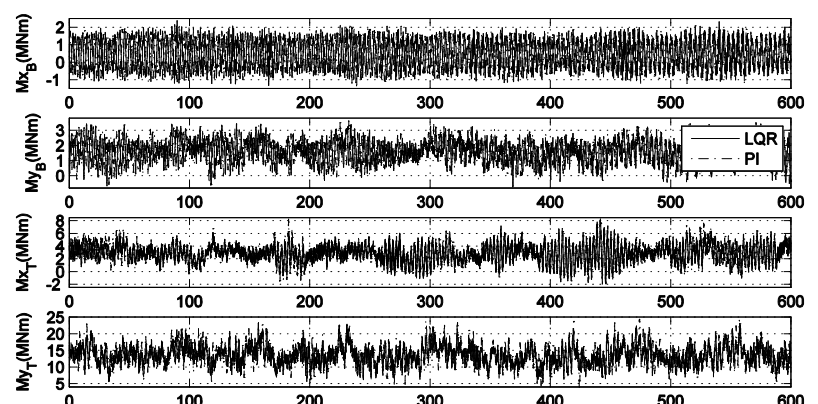

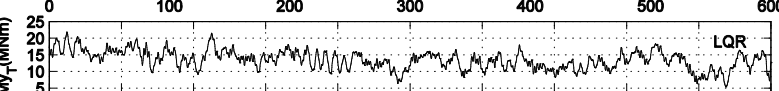

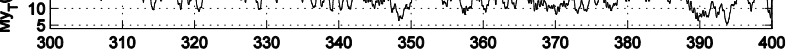

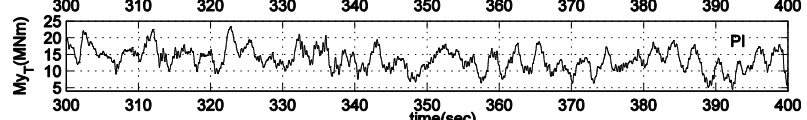

Fig. 13. Mechanical loads at wind speed of $18 \mathrm{~m} / \mathrm{s} 16.97 \%$ TI.

side-to-side direction are improved in the whole region. How much of a decrease in the mechanical loads or how much power loss, can be managed by appropriate values for the Q and R matrix in the quadratic cost function. However, there is a no direct relashionship between the performance and mechanical stresses. The proposed LQR controller is designed to get a similar performance response with a PI controller in addition to improving the tower load reduction. This design process requires some trial and errors in selecting the $\mathrm{Q}$ and $\mathrm{R}$ matrix.

\section{CONCLUSIONS}

This paper investigated the designing of a wind-scheduling linear quadratic controller with online wind speed estimation for multi-MW size wind turbines. The controller was designed by linearising the nonlinear wind turbine along the operating point trajectory. The potential of the controller was checked by simulations with GH Bladed software. The responses of the proposed method were compared with those of the conventional PI controller. The statistical data show that the proposed controller has tradeoffs between capturing energy and alleviating mechanical loads. 


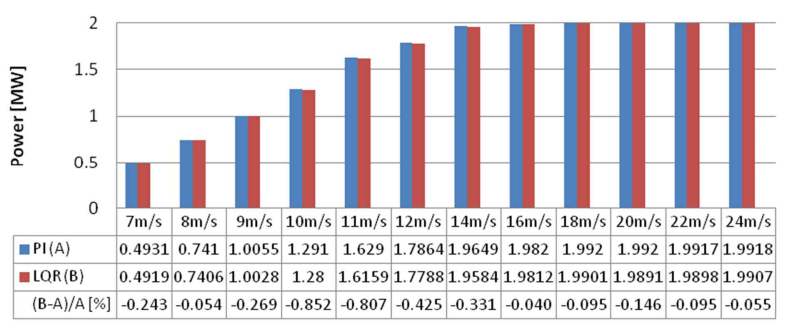

Fig. 14. Electrical power.

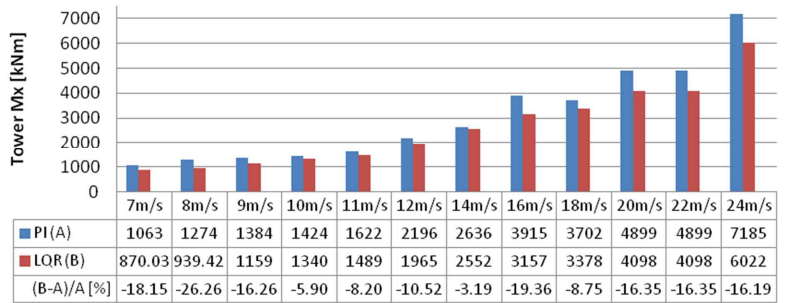

Fig. 15. Tower root bending moment in sideward direction.

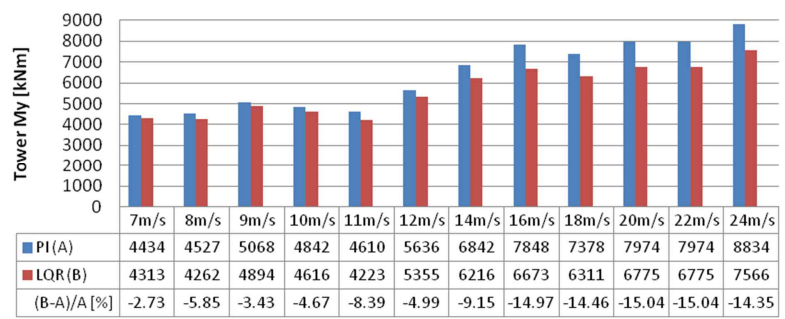

Fig. 16. Tower root bending moment in fore-aft direction.

\section{ACKNOWLEDGMENT}

This work was supported by the New \& Renewable Energy program (No. 2012T100201670) and the Human Resources Development program(No. 20134030200240) of the Korea Institute of Energy Technology Evaluation and Planning(KETEP) grant funded by the Korea government Ministry of Trade, Industry and Energy. And, this study was supported by 2013 Research Grant from Kangwon National University (No. 120131305).

\section{APPENDIX}

TABLE V

WIND TURBINE DATA

\begin{tabular}{|l|c|c|}
\hline \multicolumn{1}{|c|}{ Parameter } & Value & Unit \\
\hline Rotor diameter & 42.65 & $\mathrm{~m}$ \\
\hline Number of blades & 3 & - \\
\hline Hub height & 80 & $\mathrm{~m}$ \\
\hline Gearbox ratio & 72.28 & - \\
\hline Generator & Variable speed & - \\
\hline Cut-in wind speed & 3 & $\mathrm{~m} / \mathrm{s}$ \\
\hline Cut-out wind speed & 25 & $\mathrm{~m} / \mathrm{s}$ \\
\hline Rated wind speed & 11.3 & $\mathrm{~m} / \mathrm{s}$ \\
\hline Rated rotational speed & 15.3 & $\mathrm{rpm}$ \\
\hline Rated power & 2.0 & $\mathrm{Mw}$ \\
\hline
\end{tabular}

\section{REFERENCES}

[1] E. A. Bossanyi," "The design of closed loop controllers for wind turbines," Wind energy, Vol. 3, No. 3, pp. 148-163, Jul./Sep. 2000.

[2] K. Z. Ostergaard, P. Brath, and J. Stoustrup, "Gain-scheduling linear quadratic control of wind turbines operating at high wind speed," 16th IEEE International Conference on Control Applications, Singapore, pp. 276-281, 2007.

[3] K. Stol and M. Balas, "Full-state feedback control of a variable-Speed wind turbine: A comparison of periodic and constant gains," ASME J. Solar energy engineering, Vol.123, No. 4, pp. 319-326, Jul. 2001.

[4] E. B. Muhando, T. Senjyu, H. Kinjo, and T. Funabashi, "Augmented LQG controller for enhancement of online dynamic performance for WTG system," Renewable energy, Vol. 33,No. 8, pp. 1942-1952, Aug. 2008.

[5] E. L. van der Hooft, P. Shaak, and T. G. van Engelen, "Wind turbine control algorithms," ECN-C-03-111, 2003.

[6] Y. Nam, J. Kim, I. Paek, Y. H. Moon, S. J. Kim, and D. J. Kim, "Feedforward Pitch control using wind speed estimation," Journal of Power Electronics, Vol. 11, No. 2, pp. 211-217, Mar. 2011.

[7] K. Z. Ostergaard, P. Brath, and J. Stroustrup, "Estimation of effective wind speed," Journal of Physics: Conference Series, Vol. 75, pp. 1-9, 2007.

[8] E. A. Bossanyi, "GH Bladed theory manual(version 3.81)," Garrad Hassan and Partners, 282-BR-009, 2009.

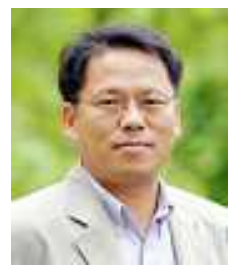

Yoonsu Nam was born in Suwon, Korea, in 1958. He received his B.S. in Nuclear Engineering from Seoul National University, Seoul, Korea, in 1981. He received his M.S. from the Department of Machine Design, Seoul National University, in 1983. He received his Ph.D. in Mechanical Engineering from Georgia Tech, Atlanta, GA, USA, in 1991. From 1983 to 1986, he was with the Central Research Institute of GoldStar (LG Electronics) Inc., Korea. From 1992 to 1997, he was with the Flight Dynamics and Control Laboratory of the Agency for Defense Development, Daejeon, Korea. In 1997 he joined the School of Mechanical and Mechatronics Engineering, Kangwon National University, Chuncheon, Korea. His current research interests include mechanical load reduction control of MW class wind turbines.

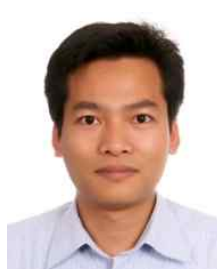

Pham Trung Kien was born in Vietnam, in 1979. He received his B.S. and M.S. from the School of Electrical Engineering, Hanoi University of Science and Technology, Hanoi, Vietnam, in 2002 and 2006, respectively. His current research interests include control system theory and design, wind turbine control system design and wind

power systems.

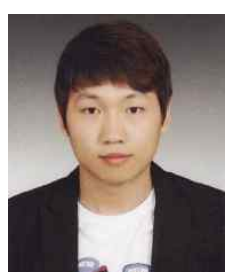

Yo-Han La was born in Gyeonggi-do, Korea in 1988. He received his B.S. in Mechanical and Mechatronics Engineering from Kangwon National University, Chuncheon, Korea, in 2011. He is currently working toward his M.S. at Kangwon National University. His current research interests include mechanical load reduction control of MW class wind turbines. 